\title{
EXISTENCE AND UNIQUENESS THEOREMS FOR SEQUENTIAL LINEAR CONFORMABLE FRACTIONAL DIFFERENTIAL EQUATIONS
}

\author{
AHMET GÖKDOĞAN, EMRAH ÜNAL, AND ERCAN ÇELIKK \\ Received 20 April, 2015
}

\begin{abstract}
Recently, a new definition of fractional derivative called as the conformable fractional derivative which based on limits introduced by Khalil et al (2014). Later, Abdeljawad (2015) improved these definitions and gave the basic concepts in this new fractional calculus. In this paper, we generalize Abel's formula and Wronskian determinant definition and establish existence and uniqueness theorems for sequential linear conformable fractional differential equations.
\end{abstract}

2010 Mathematics Subject Classification: 34A08; 34A12

Keywords: sequential linear fractional differential equations, conformable fractional derivative, existence and uniqueness theorems

\section{INTRODUCTION}

Although the idea of fractional derivative has been suggested by L'Hospital at the end 17th century, intensive studies about fractional derivative were carried out in the previous and current centuries. Integral form for fractional derivative are used by many researchers. It is known that the most popular definitions of fractional derivative are Riemann-Liouville and Caputo definitions. For Riemann-Liouville, Caputo and other definitions and the characteristics of these definitions, we refer to reader to [12],[15],[16].

(I) Riemann-Liouville definition:

$$
D_{x}^{\alpha} f(x)=\frac{1}{\Gamma(n-\alpha)}\left(\frac{d}{d x}\right)^{n} \int_{0}^{x}(x-t)^{(n-\alpha-1)} f(t) d t, n-1<\alpha \leq n
$$

(II) Caputo definition:

$$
D_{x}^{\alpha} f(x)=\frac{1}{\Gamma(n-\alpha)} \int_{0}^{x}(x-t)^{(n-\alpha-1)}\left(\frac{d}{d t}\right)^{n} f(t) d t, n-1<\alpha \leq n
$$

Recently, Khalil et al. introduced a new definition of fractional derivative and fractional integral [10]. This new definition is based on a limit form similar as in 
usual definition of derivative. Later, this new theory is improved by Abdeljawad [1]. For instance, Taylor power series representation and Laplace transform of few certain functions, fractional integration by parts formulas, chain rule and Gronwall inequality are provided by him. In addition these studies, many studies with regard to conformable fractional calculus were done [2],[3],[9].

Several studies on theory and application of the sequential linear fractional differential equations which based on Hadamard, Riemann-Liouville and Caputo derivatives were carried out [13],[5],[14],[11],[6],[4]. In this study, we generalize Abel's formula and Wronskian determinant definition [8]. Further, we investigate existence and uniqueness theorems for sequential linear conformable fractional differential equations.

\section{CONFORMABLE FRACTIONAL DERIVATIVE}

Here, we give some basic definitions and properties of the conformable fractional calculus theory which can be found in [10],[1].

Definition 1. $f:[0, \infty) \rightarrow \mathbb{R}$ let a function. Then, for all $t>0$, the conformable fractional derivative of $f$ of order $\alpha$ is defined as

$$
T_{\alpha}(f)(t)=\lim _{\varepsilon \rightarrow 0} \frac{f\left(t+\varepsilon t^{1-\alpha}\right)-f(t)}{\varepsilon}
$$

where $\alpha \in(0,1)$. If $f$ is $\alpha$-differentiable in some $(0, a), a>0$ and $\lim _{t \rightarrow 0^{+}} f^{(\alpha)}(t)$ exists, then,

$$
f^{(\alpha)}(0)=\lim _{t \rightarrow 0^{+}} f^{(\alpha)}(t)
$$

is true.

Theorem 1. let be $\alpha \in(0,1]$ and $f, g$ be $\alpha$-differentiable at a point $t>0$. Then,

(1) $T_{\alpha}(a f+b g)=a T_{\alpha}(f)+b T_{\alpha}(g)$, for all $a, b \in \mathbb{R}$,

(2) $T_{\alpha}\left(t^{p}\right)=p t^{p-\alpha}$ for all $p \in \mathbb{R}$,

(3) $T_{\alpha}(\lambda)=0$ for all constant functions $f(t)=\lambda$,

(4) $T_{\alpha}(f g)=T_{\alpha}(f) g+f T_{\alpha}(g)$,

(5) $T_{\alpha}(f / g)=\frac{T_{\alpha}(f) g-f T_{\alpha}(g)}{g^{2}}$,

(6) In addition, if $f$ is differentiable, then $T_{\alpha}(f(t))=t^{1-\alpha} \frac{d f}{d t}$.

Additionally, conformable fractional derivatives of certain functions as follow:

(i) $T_{\alpha}\left(\sin \frac{1}{\alpha} t^{\alpha}\right)=\cos \frac{1}{\alpha} t^{\alpha}$,

(ii) $T_{\alpha}\left(\cos \frac{1}{\alpha} t^{\alpha}\right)=-\sin \frac{1}{\alpha} t^{\alpha}$,

(iii) $T_{\alpha}\left(e^{\frac{1}{\alpha} t^{\alpha}}\right)=e^{\frac{1}{\alpha} t^{\alpha}}$. 
Theorem 2. Let $h(t)=f(g(t)), \alpha \in(0,1]$ and $f, g:(0, \infty) \rightarrow \mathbb{R}$ be $\alpha$-differentiable functions. Then, $h(t)$ is $\alpha$-differentiable and for all $t(t \neq 0$ and $g(t) \neq 0)$, we have

$$
T_{\alpha}(h)(t)=\left(T_{\alpha} f\right)(g(t))\left(T_{\alpha} g\right)(t) g(t)^{\alpha-1} .
$$

If $t=0$, we have

$$
T_{\alpha}(h)(t)=\lim _{t \rightarrow 0^{+}}\left(T_{\alpha} f\right)(g(t))\left(T_{\alpha} g\right)(t) g(t)^{\alpha-1} .
$$

Theorem 3. Let $f, g:[a, b] \rightarrow \mathbb{R}$ be two functions such that $f, g$ are differentiable. Then,

$$
\int_{a}^{b} f(t) T_{\alpha}(g)(t) d_{\alpha}(t)=\left.f g\right|_{a} ^{b}-\int_{a}^{b} g(t) T_{\alpha}(f)(t) d_{\alpha}(t) .
$$

\section{CONFORMABLE FRACTIONAL INTEGRAL}

Definition 2. Let be $f:[0, \infty) \rightarrow \mathbb{R}$. Then, for all $t>0, \alpha \in(0,1)$, the conformable fractional integral of $f$ of order $\alpha$ is defined by

$$
\left(I_{\alpha} f\right)(t)=\int_{0}^{t} f(x) d_{\alpha}(x)=\int_{0}^{t} x^{\alpha-1} f(x) d x
$$

where the integral means as the usual Riemann improper integral.

Theorem 4. Let $f:[0, \infty) \rightarrow \mathbb{R}$ be any continuous function and $0<\alpha \leq 1$. Then, $\forall t>a$,

$$
T_{\alpha} I_{\alpha} f(t)=f(t) .
$$

Theorem 5. Let $f:(a, b) \rightarrow \mathbb{R}$ be differentiable and $0<\alpha \leq 1$. Then, for all $t>a$,

$$
I_{\alpha} T_{\alpha} f(t)=f(t)-f(a) .
$$

\section{EXISTENCE AND UNIQUENESS THEOREMS}

Consider with consider linear sequential conformable fractional differential equations of order $n \alpha$

$$
\begin{aligned}
& { }^{n} T_{\alpha} y+p_{n-1}(t)^{n-1} T_{\alpha} y+\ldots+p_{2}(t)^{2} T_{\alpha} y+p_{1}(t) T_{\alpha} y+p_{0}(t) y=0, \\
& { }^{n} T_{\alpha} y+p_{n-1}(t)^{n-1} T_{\alpha} y+\ldots+p_{2}(t)^{2} T_{\alpha} y+p_{1}(t) T_{\alpha} y+p_{0}(t) y=f(t)
\end{aligned}
$$

where ${ }^{n} T_{\alpha} y=T_{\alpha} T_{\alpha} \ldots T_{\alpha}$. We define $n^{\text {th }}$ order differential operator as following:

$$
L_{\alpha}[y]={ }^{n} T_{\alpha} y+p_{n-1}(t)^{n-1} T_{\alpha} y+\ldots+p_{2}(t)^{2} T_{\alpha} y+p_{1}(t) T_{\alpha} y+p_{0}(t) y .
$$


Theorem 6. Let $t^{\alpha-1} p(t), t^{\alpha-1} q(t) \in C(a, b)$ and $y$ be $\alpha$-differentiable for $0<$ $\alpha \leq 1$. Then, the following initial value problem

$$
\begin{gathered}
T_{\alpha} y+p(t) y=q(t), \\
y\left(t_{0}\right)=y_{0}
\end{gathered}
$$

has a unique solution on the interval $(a, b)$ for any $t_{0} \in(a, b)$.

Proof. we can write

$$
\begin{gathered}
T_{\alpha} y+p(t) y=q(t), \\
t^{1-\alpha} y^{\prime}+p(t) y=q(t), \\
y^{\prime}+t^{\alpha-1} p(t) y=t^{\alpha-1} q(t) .
\end{gathered}
$$

So, existence and uniqueness follows from classical theorem for linear equation.

Theorem 7. Let $t^{\alpha-1} p_{n-1}(t), \ldots, t^{\alpha-1} p_{1}(t), t^{\alpha-1} p_{0}(t), t^{\alpha-1} q(t) \in C(a, b)$ and let $y$ be $n$ times $\alpha$-differentiable function. Then, a solution $y(t)$ of the next initial value problem

$$
\begin{gathered}
{ }^{n} T_{\alpha} y+p_{n-1}(t)^{n-1} T_{\alpha} y+\ldots+p_{2}(t)^{2} T_{\alpha} y+p_{1}(t) T_{\alpha} y+p_{0}(t) y=q(t), \\
y\left(t_{0}\right)=y_{0}, T_{\alpha} y\left(t_{0}\right)=y_{1}, \ldots,{ }^{n-1} T_{\alpha} y\left(t_{0}\right)=y_{n-1}, \quad a<t_{0}<b
\end{gathered}
$$

exists on the interval $[a, b]$ and it is unique.

Proof. To show existence of a local solution, we reduce our problem to the first order system of differential equations. So, let's make the following change of variables

$$
x_{1}=y, x_{2}=T_{\alpha} y, x_{3}={ }^{2} T_{\alpha} y, \cdots, x_{n}={ }^{n-1} T_{\alpha} y\left(t_{0}\right)=y_{n-1} .
$$

Hence, we have

$$
\begin{aligned}
T_{\alpha} x_{1} & =x_{2}, \\
T_{\alpha} x_{2} & =x_{3}, \\
& \vdots \\
T_{\alpha} x_{n-1} & =x_{n}, \\
T_{\alpha} x_{n} & =-p_{n-1} x_{n}-\cdots-p_{2} x_{3}-p_{1} x_{2}-p_{0} x_{1}+q(t) .
\end{aligned}
$$


Now, we can rewrite the problem in the following form

$$
\underbrace{T_{\alpha}\left[\begin{array}{c}
x_{1} \\
x_{2} \\
\vdots \\
x_{n-1} \\
x_{n}
\end{array}\right]}_{X(t)}+\underbrace{\left[\begin{array}{cccccc}
0 & -1 & 0 & 0 & \cdots & 0 \\
0 & 0 & -1 & 0 & \cdots & 0 \\
\vdots & \vdots & \vdots & \vdots & \vdots & \vdots \\
0 & 0 & 0 & 0 & \cdots & -1 \\
p_{0} & p_{1} & p_{2} & p_{3} & \cdots & p_{n-1}
\end{array}\right]}_{P(t)} \underbrace{\left[\begin{array}{c}
x_{1} \\
x_{2} \\
\vdots \\
x_{n-1} \\
x_{n}
\end{array}\right]}_{X^{\prime}(t)+t^{\alpha-1} P(t) X(t)=t^{\alpha-1} Q(t) .}=\underbrace{\left[\begin{array}{c}
0 \\
0 \\
\vdots \\
0 \\
q(t)
\end{array}\right]}_{Q(t)}
$$

Finally, the existence and uniqueness of solution (4.9)-(4.10) follows from classical theorem on existence and uniqueness for system equation [7].

Theorem 8. $L_{\alpha}$ is linear, i.e.

$$
L_{\alpha}\left[c_{1} y_{1}+c_{2} y_{2}\right]=c_{1} L_{\alpha}\left[y_{1}\right]+c_{2} L_{\alpha}\left[y_{2}\right]
$$

where $y_{1}, y_{2}$ are $n$ times $\alpha$-differentiable functions and $c_{1}, c_{2}$ are arbitrary numbers.

Proof. From the definition conformable fractional derivative it follows that

$$
\begin{aligned}
L_{\alpha}\left[c_{1} y_{1}+c_{2} y_{2}\right] & ={ }^{n} T_{\alpha}\left(c_{1} y_{1}+c_{2} y_{2}\right)+p_{n-1}(t)^{n-1} T_{\alpha}\left(c_{1} y_{1}+c_{2} y_{2}\right)+\cdots \\
& +p_{1}(t) T_{\alpha}\left(c_{1} y_{1}+c_{2} y_{2}\right)+p_{0}(t)\left(c_{1} y_{1}+c_{2} y_{2}\right) \\
& =c_{1}\left({ }^{n} T_{\alpha} y_{1}+p_{n-1}(t)^{n-1} T_{\alpha} y_{1}+\ldots+p_{0}(t) y_{1}\right) \\
& +c_{2}\left({ }^{n} T_{\alpha} y_{2}+p_{n-1}(t)^{n-1} T_{\alpha} y_{2}+\ldots+p_{0}(t) y_{2}\right) .
\end{aligned}
$$

So, we have

$$
L_{\alpha}\left[c_{1} y_{1}+c_{2} y_{2}\right]=c_{1} L_{\alpha}\left[y_{1}\right]+c_{2} L_{\alpha}\left[y_{2}\right] .
$$

Theorem 9. let $y_{1}, y_{2}, \ldots, y_{n}$ be the solutions of the equation $L_{\alpha}[y]=0$. In this case, the linear combination

$$
y=c_{1} y_{1}+c_{2} y_{2}+\cdots+c_{n} y_{n}
$$

is also its solution for the arbitrary constants $c_{k}, k=1, \ldots, n$.

Proof. Let $y_{1}(t), y_{2}(t), \ldots, y_{n}(t)$ be solutions of the equation $L_{\alpha}[y]=0$, for arbitrary constants $c_{k}, k=1, \ldots, n$. Denote by

$$
y=c_{1} y_{1}+c_{2} y_{2}+\cdots+c_{n} y_{n} .
$$

By using linear property of $L_{\alpha}$, we have

$$
L_{\alpha}(y)=L_{\alpha}\left(c_{1} y_{1}+c_{2} y_{2}+\cdots+c_{n} y_{n}\right)
$$




$$
\begin{aligned}
& =c_{1} L_{\alpha}\left(y_{1}\right)+c_{2} L_{\alpha}\left(y_{2}\right)+\cdots+c_{n} L_{\alpha}\left(y_{n}\right) \\
& =0 .
\end{aligned}
$$

Definition 3. We assume that $y_{1}(t), y_{2}(t), \ldots, y_{n}(t)$ are at least $(n-1)$ times $\alpha$-differentiable functions. For any $0<\alpha \leq 1$, the determinant

$$
W_{\alpha}\left(y_{1}, y_{2}, \ldots, y_{n}\right)=\left|\begin{array}{cccc}
y_{1} & y_{2} & \ldots & y_{n} \\
T_{\alpha} y_{1} & T_{\alpha} y_{2} & \ldots & T_{\alpha} y_{n} \\
\vdots & \vdots & \ldots & \vdots \\
{ }^{n-1} T_{\alpha} y_{1} & { }^{n-1} T_{\alpha} y_{2} & \ldots & { }^{n-1} T_{\alpha} y_{n}
\end{array}\right|
$$

is called $\alpha-$ Wronskian of the functions.

Definition 4. Any set $\left\{y_{1}, y_{2}, \ldots, y_{n}\right\}$ of n solutions of $L_{\alpha}[y]=0$ is said to be a fundamental set of solutions if every solution of this equation can be written as a linear combination of these functions.

Theorem 10. Let $y_{1}, y_{2}, \ldots, y_{n}$ be $n$ solutions of $L_{\alpha}[y]=0$. If there is a $t_{0} \in$ $(a, b)$ such that $W_{\alpha}\left(y_{1}, y_{2}, \ldots, y_{n}\right)\left(t_{0}\right) \neq 0$, then, $\left\{y_{1}, y_{2}, \ldots, y_{n}\right\}$ is a fundamental set of solutions.

Proof. If $y(t)$ is a solution of $L_{\alpha}[y]=0$, then we can write $y(t)$ as a linear combination of $y_{1}, y_{2}, \ldots, y_{n}$ which we need to show. That is

$$
y=c_{1} y_{1}+c_{2} y_{2}+\cdots+c_{n} y_{n} .
$$

So, the problem is reduced to finding the constants $c_{k}, 1 \leq k \leq n$. We can write the following linear system of equations

$$
\begin{gathered}
c_{1} y_{1}\left(t_{0}\right)+c_{2} y_{2}\left(t_{0}\right)+\cdots+c_{n} y_{n}\left(t_{0}\right)=y\left(t_{0}\right) \\
c_{1} T_{\alpha} y_{1}\left(t_{0}\right)+c_{2} T_{\alpha} y_{2}\left(t_{0}\right)+\cdots+c_{n} T_{\alpha} y_{n}\left(t_{0}\right)=T_{\alpha} y\left(t_{0}\right) \\
\vdots \\
c_{1}{ }^{n-1} T_{\alpha} y_{1}\left(t_{0}\right)+c_{2}{ }^{n-1} T_{\alpha} y_{2}\left(t_{0}\right)+\cdots+c_{n}{ }^{n-1} T_{\alpha} y_{n}\left(t_{0}\right) \\
={ }^{n-1} T_{\alpha} y\left(t_{0}\right) .
\end{gathered}
$$

Applying Cramer's rule, we can get

$$
c_{k}=\frac{W_{\alpha}^{k}\left(t_{0}\right)}{W_{\alpha}\left(t_{0}\right)}, \quad 1 \leq k \leq n .
$$

Since $W_{\alpha}\left(t_{0}\right) \neq 0$, it follows that $c_{1}, c_{2}, \ldots, c_{n}$ exist.

Theorem 11. Suppose that $t^{\alpha-1} p_{n-1}(t), \ldots, t^{\alpha-1} p_{1}(t), t^{\alpha-1} p_{0}(t) \in C(a, b)$. Then, the equation $L_{\alpha}[y]=0$ has a fundamental set of solutions $\left\{y_{1}, y_{2}, \ldots, y_{n}\right\}$. 
Proof. Let $t_{0} \in(a, b)$. Consider the following $\mathrm{n}$ initial value problems

$$
\begin{aligned}
& L_{\alpha}[y]=0, y\left(t_{0}\right)=1, T_{\alpha} y\left(t_{0}\right)=0, \ldots,{ }^{n-1} T_{\alpha} y\left(t_{0}\right)=0 \\
& L_{\alpha}[y]=0, y\left(t_{0}\right)=0, T_{\alpha} y\left(t_{0}\right)=1, \ldots,{ }^{n-1} T_{\alpha} y\left(t_{0}\right)=0 \\
& L_{\alpha}[y]=0, y\left(t_{0}\right)=0, T_{\alpha} y\left(t_{0}\right)=0, \ldots,{ }^{n-1} T_{\alpha} y\left(t_{0}\right)=1
\end{aligned}
$$

From 7, it follows that there is the solution $y_{i}$ of $i^{\text {th }}$ problem for each index $i$. By the Theorem 10, the set of $\left\{y_{1}, y_{2}, \ldots, y_{n}\right\}$ is a fundamental set of solutions since

$$
W_{\alpha}(t)=\left|\begin{array}{cccc}
1 & 0 & \cdots & 0 \\
0 & 1 & \cdots & 0 \\
\vdots & \vdots & \cdots & \vdots \\
0 & 0 & \cdots & 1
\end{array}\right|=1 \neq 0
$$

Theorem 12. Let $y_{1}, y_{2}, \ldots, y_{n}$ be $n$ solutions of equation $L_{\alpha}[y]=0$. Then

(1) $W_{\alpha}(t)$ satisfies the differential equation $T_{\alpha} W_{\alpha}+p_{n-1}(t) W_{\alpha}=0$,

(2) For any $t_{0} \in(a, b)$

$$
W_{\alpha}(t)=W_{\alpha}\left(t_{0}\right) e^{-\int_{t_{0}}^{t} x^{\alpha-1} p_{n-1}(x) d x} .
$$

Moreover, if $W_{\alpha}\left(t_{0}\right) \neq 0$ then, $W_{\alpha}(t) \neq 0$ for all $t \in(a, b)$.

Proof. Introduce new variables

$$
x_{1}=y, x_{2}=T_{\alpha} y, x_{3}={ }^{2} T_{\alpha} y, \cdots, x_{n}={ }^{n-1} T_{\alpha} y\left(t_{0}\right)=y_{n-1} .
$$

We can rewrite the differential in the matrix form

$$
T_{\alpha} \underbrace{\left[\begin{array}{c}
x_{1} \\
x_{2} \\
\vdots \\
x_{n-1} \\
x_{n}
\end{array}\right]}_{X(t)}=\underbrace{\left[\begin{array}{cccccc}
0 & 1 & 0 & 0 & \cdots & 0 \\
0 & 0 & 1 & 0 & \cdots & 0 \\
\vdots & \vdots & \vdots & \vdots & \vdots & \vdots \\
0 & 0 & 0 & 0 & \cdots & 1 \\
-p_{0} & -p_{1} & -p_{2} & -p_{3} & \cdots & -p_{n-1}
\end{array}\right]}_{P(t)} \underbrace{\left[\begin{array}{c}
T_{\alpha} X(t)=P(t) X(t) . \\
x_{1} \\
x_{2} \\
\vdots \\
x_{n-1} \\
x_{n}
\end{array}\right]}_{X(t)},
$$

Therefore, we obtain

$$
T_{\alpha} W_{\alpha}(t)=\left(p_{11}+p_{22}+\cdots+p_{n n}\right) W_{\alpha}(t) .
$$

Really, if $\alpha$-conformable derivative of $W_{\alpha}\left(y_{1}, y_{2}, \ldots, y_{n}\right)$ is calculated, then, we can get

$$
T_{\alpha} W_{\alpha}(t)=-p_{n-1}(t) W_{\alpha}(t) .
$$


Hence,

$$
\begin{gathered}
\frac{T_{\alpha} W_{\alpha}(t)}{W_{\alpha}(t)}=-p_{n-1}(t), \\
\ln \left(W_{\alpha}(t)\right)-\ln \left(W_{\alpha}\left(t_{0}\right)\right)=-\int_{t_{0}}^{t} x^{\alpha-1} p_{n-1}(x) d x, \\
W_{\alpha}(t)=W_{\alpha}\left(t_{0}\right) e^{-\int_{t_{0}}^{t} x^{\alpha-1} p_{n-1}(x) d x} .
\end{gathered}
$$

So, proof of theorem is finished.

Theorem 13. Let $t^{\alpha-1} p_{n-1}(t), \ldots, t^{\alpha-1} p_{1}(t), t^{\alpha-1} p_{0}(t) \in C(a, b)$. If $\left\{y_{1}, y_{2}, \ldots, y_{n}\right\}$ is a fundamental set of solutions of $L_{\alpha}[y]=0$, then, $W_{\alpha}(t) \neq 0$ for all $t \in(a, b)$.

Proof. Suppose that $t_{0}$ be any point in $(a, b)$. By Theorem 7 , there is a unique solution $y(t)$ of the initial value problem

$$
L_{\alpha}[y]=0, y\left(t_{0}\right)=1, T_{\alpha} y\left(t_{0}\right)=0, \ldots,{ }^{n-1} T_{\alpha} y\left(t_{0}\right)=0 .
$$

There exist unique constants $c_{1}, c_{2}, \ldots, c_{n}$ such that

$$
\begin{aligned}
c_{1} y_{1}(t)+c_{2} y_{2}(t)+\cdots+c_{n} y_{n}(t) & =y(t), \\
c_{1} T_{\alpha} y_{1}(t)+c_{2} T_{\alpha} y_{2}(t)+\cdots+c_{n} T_{\alpha} y_{n}(t) & =T_{\alpha} y(t), \\
\vdots & \\
c_{1}{ }^{n-1} T_{\alpha} y_{1}(t)+c_{2}{ }^{n-1} T_{\alpha} y_{2}(t)+\cdots+c_{n}{ }^{n-1} T_{\alpha} y_{n}(t) & ={ }^{n-1} T_{\alpha} y(t)
\end{aligned}
$$

for all $t \in(a, b)$ since $\left\{y_{1}, y_{2}, \ldots, y_{n}\right\}$ is a fundamental set of solutions. In particular, for $t=t_{0}$, we obtain the system

$$
\begin{aligned}
c_{1} y_{1}\left(t_{0}\right)+c_{2} y_{2}\left(t_{0}\right)+\cdots+c_{n} y_{n}\left(t_{0}\right) & =1, \\
c_{1} T_{\alpha} y_{1}\left(t_{0}\right)+c_{2} T_{\alpha} y_{2}\left(t_{0}\right)+\cdots+c_{n} T_{\alpha} y_{n}\left(t_{0}\right) & =0, \\
& \vdots \\
c_{1}{ }^{n-1} T_{\alpha} y_{1}\left(t_{0}\right)+c_{2}{ }^{n-1} T_{\alpha} y_{2}\left(t_{0}\right)+\cdots+c_{n}{ }^{n-1} T_{\alpha} y_{n}\left(t_{0}\right) & =0 .
\end{aligned}
$$

This system has a unique solution

$$
c_{k}=\frac{W_{\alpha}^{k}}{W_{\alpha}\left(t_{0}\right)}, \quad 1 \leq k \leq n .
$$

Here, for every $k$

$$
\begin{gathered}
\multicolumn{10}{c}{W_{\alpha}^{k}=} \\
{\left[\begin{array}{cccccccc}
y_{1}\left(t_{0}\right) & y_{2}\left(t_{0}\right) & \ldots & y_{k-1}\left(t_{0}\right) & 1 & y_{k}\left(t_{0}\right) & \ldots & y_{n}\left(t_{0}\right) \\
T_{\alpha} y_{1}\left(t_{0}\right) & T_{\alpha} y_{2}\left(t_{0}\right) & \ldots & T_{\alpha} y_{k-1}\left(t_{0}\right) & 0 & T_{\alpha} y_{k}\left(t_{0}\right) & \ldots & T_{\alpha} y_{n}\left(t_{0}\right) \\
\vdots & \vdots & \vdots & \vdots & \vdots & \vdots & \vdots & \vdots \\
{ }^{n-1} T_{\alpha} y_{1}\left(t_{0}\right) & { }^{n-1} T_{\alpha} y_{2}\left(t_{0}\right) & \ldots & { }^{n-1} T_{\alpha} y_{k-1}\left(t_{0}\right) & 0 & { }^{n-1} T_{\alpha} y_{k}\left(t_{0}\right) & \ldots & { }^{n-1} T_{\alpha} y_{n}\left(t_{0}\right)
\end{array}\right] .}
\end{gathered}
$$


For existence $c_{1}, c_{2}, \ldots, c_{n}$ it should be $W_{\alpha}\left(t_{0}\right) \neq 0$. By Theorem 12, we conclude that $W_{\alpha}\left(t_{0}\right) \neq 0$ for all $t_{0} \in(a, b)$.

Theorem 14. Let $t^{\alpha-1} p_{n-1}(t), \ldots, t^{\alpha-1} p_{1}(t), t^{\alpha-1} p_{0}(t) \in C(a, b)$. The solution set $\left\{y_{1}, y_{2}, \ldots, y_{n}\right\}$ is a fundamental set of solutions of equation $L_{\alpha}[y]=0$ if and only if the functions $y_{1}, y_{2}, \ldots, y_{n}$ are linearly independent.

Proof. Suppose that $\left\{y_{1}, y_{2}, \ldots, y_{n}\right\}$ is a fundamental set of solutions, then by Theorem 13 it follows that there is $t_{0} \in(a, b)$ such that $W_{\alpha}\left(t_{0}\right) \neq 0$. Assume that

$$
c_{1} y_{1}(t)+c_{2} y_{2}(t)+\cdots+c_{n} y_{n}(t)=0
$$

for all $t \in(a, b)$. By repeated $\alpha$-differentiation of the equation (4.40), we find

$$
\begin{aligned}
c_{1} y_{1}(t)+c_{2} y_{2}(t)+\cdots+c_{n} y_{n}(t) & =0, \\
c_{1} T_{\alpha} y_{1}(t)+c_{2} T_{\alpha} y_{2}(t)+\cdots+c_{n} T_{\alpha} y_{n}(t) & =0, \\
& \vdots \\
c_{1}{ }^{n-1} T_{\alpha} y_{1}(t)+c_{2}{ }^{n-1} T_{\alpha} y_{2}(t)+\cdots+c_{n}{ }^{n-1} T_{\alpha} y_{n}(t) & =0 .
\end{aligned}
$$

Thus, one finds $c_{1}, c_{2}, \ldots, c_{n}$ by solving the system

$$
\begin{aligned}
c_{1} y_{1}\left(t_{0}\right)+c_{2} y_{2}\left(t_{0}\right)+\cdots+c_{n} y_{n}\left(t_{0}\right) & =0, \\
c_{1} T_{\alpha} y_{1}\left(t_{0}\right)+c_{2} T_{\alpha} y_{2}\left(t_{0}\right)+\cdots+c_{n} T_{\alpha} y_{n}\left(t_{0}\right) & =0, \\
& \vdots \\
c_{1}{ }^{n-1} T_{\alpha} y_{1}\left(t_{0}\right)+c_{2}{ }^{n-1} T_{\alpha} y_{2}\left(t_{0}\right)+\cdots+c_{n}{ }^{n-1} T_{\alpha} y_{n}\left(t_{0}\right) & =0 .
\end{aligned}
$$

Namely, by using Cramer's rule one finds

$$
c_{1}=c_{2}=\ldots=c_{n}=\frac{0}{W_{\alpha}\left(t_{0}\right)}=0
$$

Thus, set of functions $\left\{y_{1}, y_{2}, \ldots, y_{n}\right\}$ are linearly independent. Contrarily, suppose $\left\{y_{1}, y_{2}, \ldots, y_{n}\right\}$ is a linearly independent set and suppose

$\left\{y_{1}, y_{2}, \ldots, y_{n}\right\}$ is not a fundamental set of solutions. Then, by Theorem 10, we get $W_{\alpha}(t)=0$, for all $t \in(a, b)$. We choose any $t_{0} \in(a, b)$. Then $W_{\alpha}\left(t_{0}\right)=0$. But, this says $W_{\alpha}\left(t_{0}\right) \neq 0$ that the matrix

$$
\left[\begin{array}{cccc}
y_{1}\left(t_{0}\right) & y_{2}\left(t_{0}\right) & \ldots & y_{n}\left(t_{0}\right) \\
T_{\alpha} y_{1}\left(t_{0}\right) & T_{\alpha} y_{2}\left(t_{0}\right) & \ldots & T_{\alpha} y_{n}\left(t_{0}\right) \\
\vdots & \vdots & \ldots & \vdots \\
{ }^{n-1} T_{\alpha} y_{1}\left(t_{0}\right) & { }^{n-1} T_{\alpha} y_{2}\left(t_{0}\right) & \ldots & { }^{n-1} T_{\alpha} y_{n}\left(t_{0}\right)
\end{array}\right]
$$


is not invertible which means that there exist $c_{1}, c_{2}, \ldots, c_{n}, c_{1}^{2}+c_{2}^{2}+\ldots+c_{n}^{2} \neq 0$ such that

$$
\begin{aligned}
c_{1} y_{1}\left(t_{0}\right)+c_{2} y_{2}\left(t_{0}\right)+\cdots+c_{n} y_{n}\left(t_{0}\right) & =0, \\
c_{1} T_{\alpha} y_{1}\left(t_{0}\right)+c_{2} T_{\alpha} y_{2}\left(t_{0}\right)+\cdots+c_{n} T_{\alpha} y_{n}\left(t_{0}\right) & =0, \\
& \vdots \\
c_{1}{ }^{n-1} T_{\alpha} y_{1}\left(t_{0}\right)+c_{2}{ }^{n-1} T_{\alpha} y_{2}\left(t_{0}\right)+\cdots+c_{n}{ }^{n-1} T_{\alpha} y_{n}\left(t_{0}\right) & =0 .
\end{aligned}
$$

Now, let

$$
y(t)=c_{1} y_{1}(t)+c_{2} y_{2}(t)+\cdots+c_{n} y_{n}(t)
$$

for all $t \in(a, b)$. Then, $y(t)$ is the solution of the differential equation and

$$
y\left(t_{0}\right)=T_{\alpha} y\left(t_{0}\right)=\cdots={ }^{n-1} T_{\alpha} y\left(t_{0}\right)=0 .
$$

But, the zero function is also the solution of the initial value problem. By Theorem 7 , it should be

$$
c_{1} y_{1}(t)+c_{2} y_{2}(t)+\cdots+c_{n} y_{n}(t)=0
$$

for all $t \in(a, b)$ with $c_{1}, c_{2}, \ldots, c_{n}$ not all equal to zero which means that $y_{1}, y_{2}, \ldots, y_{n}$ are linearly dependent which is not in agreement with our earlier assumption that $y_{1}, y_{2}, \ldots, y_{n}$ are linearly independent.

Theorem 15. Let $y_{1}, y_{2}, \ldots, y_{n}$ be linearly independent solutions of the equation $L_{\alpha}[y]=0$. Then, the general solution of the equation is

$$
y=c_{1} y_{1}+c_{2} y_{2}+\cdots+c_{n} y_{n}
$$

where the arbitrary constants $c_{k}, k=1, \ldots, n$.

Proof. Particular solution at any $t=t_{0}$ is obtained by the help of initial conditions as following:

$$
y\left(t_{0}\right)=\gamma_{0}, T_{\alpha} y\left(t_{0}\right)=\gamma_{1}, \cdots,{ }^{n-1} T_{\alpha} y\left(t_{0}\right)=\gamma_{n-1}
$$

where $t_{0} \in(a, b)$ and $\gamma_{0}, \gamma_{1}, \ldots \gamma_{n-1}$ are arbitrary constants. If we choose $c_{1}, c_{2}, \ldots, c_{n}$ constants to protect conditions (4.50), then, proof is completed. To accomplish this, we can write following system of equations:

$$
\begin{aligned}
c_{1} y_{1}\left(t_{0}\right)+c_{2} y_{2}\left(t_{0}\right)+\cdots+c_{n} y_{n}\left(t_{0}\right) & =\gamma_{0}, \\
c_{1} T_{\alpha} y_{1}\left(t_{0}\right)+c_{2} T_{\alpha} y_{2}\left(t_{0}\right)+\cdots+c_{n} T_{\alpha} y_{n}\left(t_{0}\right) & =\gamma_{1}, \\
& \vdots \\
c_{1}{ }^{n-1} T_{\alpha} y_{1}\left(t_{0}\right)+c_{2}{ }^{n-1} T_{\alpha} y_{2}\left(t_{0}\right)+\cdots+c_{n}{ }^{n-1} T_{\alpha} y_{n}\left(t_{0}\right) & =\gamma_{n-1} .
\end{aligned}
$$


Writing the above system in matrix form, we get

$$
\left[\begin{array}{cccc}
y_{1}\left(t_{0}\right) & y_{2}\left(t_{0}\right) & \ldots & y_{n}\left(t_{0}\right) \\
T_{\alpha} y_{1}\left(t_{0}\right) & T_{\alpha} y_{2}\left(t_{0}\right) & \ldots & T_{\alpha} y_{n}\left(t_{0}\right) \\
\vdots & \vdots & \ldots & \vdots \\
{ }^{n-1} T_{\alpha} y_{1}\left(t_{0}\right) & { }^{n-1} T_{\alpha} y_{2}\left(t_{0}\right) & \ldots & { }^{n-1} T_{\alpha} y_{n}\left(t_{0}\right)
\end{array}\right]\left[\begin{array}{c}
c_{1} \\
c_{2} \\
\vdots \\
c_{n}
\end{array}\right]=\left[\begin{array}{c}
\gamma_{0} \\
\gamma_{1} \\
\vdots \\
\gamma_{n-1}
\end{array}\right]
$$

$y_{1}, y_{2}, \ldots, y_{n}$ are linearly independent solutions of $L_{\alpha}[y]=0$, so $W_{\alpha}\left(t_{0}\right) \neq 0$. In this case, according to the fundamental theorem of algebra, system (4.51) has a unique solution. Hence, proof is completed.

Theorem 16. Let $y_{p}$ be any particular solution of the nonhomogeneous linear $n^{\text {th }}$ order sequential differential equation (4.2) and $\left\{y_{1}, y_{2}, \ldots, y_{n}\right\}$ be a fundamental set of solutions of the associated homogeneous differential equation (4.1). Then, the general solution of the equation is

$$
y=c_{1} y_{1}+c_{2} y_{2}+\cdots+c_{n} y_{n}+y_{p}
$$

where the arbitrary constants $c_{k}, k=1, \ldots, n$.

Proof. Let $L_{\alpha}$ be the differential operator and let $Y(t)$ and $y_{p}(t)$ be particular solutions of the nonhomogeneous equation $L_{\alpha}[y]=q(t)$. If we define $u(t)=$ $Y(t)-y_{p}(t)$, then, by linearity of $L_{\alpha}$, we have

$$
L_{\alpha}[u]=L_{\alpha}\left[Y(t)-y_{p}(t)\right]=L_{\alpha}[Y(t)]-L_{\alpha}\left[y_{p}(t)\right]=q(t)-q(t)=0 .
$$

This shows that $u(t)$ is a solution of the homogeneous equation $L_{\alpha}[y]=0$. Hence, by Theorem 9 ,

$$
u(t)=c_{1} y_{1}(t)+c_{2} y_{2}(t)+\cdots+c_{n} y_{n}(t)
$$

and so

$$
Y(t)-y_{p}(t)=c_{1} y_{1}(t)+c_{2} y_{2}(t)+\cdots+c_{n} y_{n}(t)
$$

or

$$
Y(t)=c_{1} y_{1}(t)+c_{2} y_{2}(t)+\cdots+c_{n} y_{n}(t)+y_{p}(t)
$$

\section{Conclusion}

In our work, we generalize Abel's formula and Wronskian determinant and present existence and uniqueness theorems for sequential linear conformable fractional differential equations. It has been found that results obtained from this work is analogous to the results obtained from the ordinary case. 


\section{REFERENCES}

[1] T. Abdeljawad, "On conformable fractional calculus," Journal of Computational and Applied Mathematics, vol. 279, pp. 57-66, 2015, doi: 10.1016/j.cam.2014.10.016.

[2] T. Abdeljawad, M. AL Horani, and R. Khalil, "Conformable fractional semigroups of operators," Journal of Semigroup Theory and Applications, vol. 2015, 2015.

[3] D. Anderson and R. Avery, "Fractional-order boundary value problem with Sturm-Liouville boundary conditions," Electronic Journal of Differential Equations, vol. 2015, no. 29, pp. 1-10, 2015.

[4] B. Bonilla, M. Rivero, and J. Trujillo, "Linear differential equations of fractional order," In Advances in fractional calculus, pp. 77-91, 2007.

[5] B. Bonilla, M. Rivero, and J. Trujillo, "On systems of linear fractional differential equations with constant coefficients," Applied Mathematics and Computation, vol. 187(1), pp. 68-78, 2007, doi: 10.1016/j.amc.2006.08.104.

[6] M. Błasik, "Numerical scheme for a two-term sequential fractional differential equation," Scientific Research of the Institute of Mathematics and Computer Science, vol. 10, pp. 17-29, 2011.

[7] M. Finan, A second course in Elementary Ordinary Differential Equations, Arkansas Tech University, 2013.

[8] M. Hammad and R. Khalil, "Abel's formula and Wronskian for conformable fractional differential equations," International Journal of Differential Equations and Applications, vol. 13, no. 2, pp. 177-183, 2014.

[9] R. Khalil, "Fractional fourier series with applications," American Journal of Computational and Applied Mathematics, vol. 4(6), pp. 187-191, 2014.

[10] R. Khalil, M. Al Horani, A. Yousef, and M. Sababheh, "A new definition of fractional derivative," Journal of Computational and Applied Mathematics, vol. 264, pp. 65-70, 2014, doi: 10.1016/j.cam.2014.01.002.

[11] A. Kilbas, M. Rivero, L. Rodríguez-Germá, and J. Trujillo, " $\alpha$-analytic solutions of some linear fractional differential equations with variable coefficients," Applied mathematics and computation, vol. 187, no. 1, pp. 239-249, 2007, doi: 10.1016/j.amc.2006.08.121.

[12] A. Kilbas, H. Srivastava, and J. Trujillo, Theory and applications of fractional differential equations. London: Elsevier Science Limited, 2006, vol. 204.

[13] M. Klimek, "Sequential fractional differential equations with Hadamard derivative," Communications in Nonlinear Science and Numerical Simulation, vol. 16(12), pp. 4689-4697, 2011, doi: 10.1016/j.cnsns.2011.01.018.

[14] G. Loghmani and S. Javanmardi, "Numerical methods for sequential fractional differential equations for Caputo operator," Bull. Malays. Math. Sci. Soc., vol. (2),35(2), pp. 315-323, 2011.

[15] K. Miller and B. Ross, An introduction to the fractional calculus and fractional differential equations. New York: A Wiley-Interscience Publication, 1993.

[16] I. Podlubny, Fractional differential equations: an introduction to fractional derivatives, fractional differential equations, to methods of their solution and some of their applications. London: Academic press, 1998.

Authors' addresses

Ahmet Gökdoğan

Gümüşhane University, Faculty Of Engineering, Department Of Mathematical Engineering, 29100 Gümüşhane, Turkey

E-mail address: gokdogan@gumushane.edu.tr 


\section{Emrah Ünal}

Artvin Çoruh University, Faculty Of Education, Department of Elementary Mathematics Education, 08100 Artvin, Turkey

E-mail address: emrah.unaleartvin.edu.tr

\section{Ercan Çelik}

Atatürk University, Faculty Of Science, Department of Mathematics, 25400 Erzurum, Turkey

E-mail address: ercelikeatauni.edu.tr 